\title{
Time Stamp based Routing Protocol for Mobile Ad-Hoc Networks (TSBR)
}

\author{
Muzzammil Hussain \\ Lecturer \\ Department of Informatics, \\ Kakatiya University, \\ Warangal, India
}

\author{
Dr A Vinaya Babu \\ Professor \\ Department of CSE, JNTU, \\ Hyderabad, India
}

\author{
Dr A Sadanandam \\ Professor \\ Department of Informatics, \\ Kakatiya University, \\ Warangal, India
}

\begin{abstract}
In this paper we propose a new routing protocol TSBR (Time Stamp Based Routing) for wireless ad hoc networks. TSBR is on-demand/reactive protocol, that discovers a route/path between source node $\mathrm{S}$ and destination node $\mathrm{D}$ when needed, the discovered path is stored in the route table of $S$ along with a time stamp value T. TSBR protocol eliminates the problem of sparse routes between nodes $\mathrm{S}$ and $\mathrm{D}$ using time stamp value $T$. the route selected for transmitting data packets by TSBR is shortest and takes less time for delivery of packets at destination node.
\end{abstract}

Keywords: Ad hoc networks, on-demand/reactive protocol, time stamp value.

\section{INTRODUCTION}

An Ad hoc network is a collection of mobile nodes forming an instant network without fixed topology [1] [3] \& [4]. These networks do not have any base infrastructure as in conventional networks. Ad hoc networks have been proposed to support scenarios where no fixed infrastructure exists. They can be set up quickly where the existing infrastructure does not meet application requirements for the reasons such as security, cost or quality. Examples for applications of Ad hoc networks range from military operations, emergency disaster relief, community networking, inter action between attendees at a meeting or students during a lecture.

Ad hoc networks differ significantly from conventional networks in the dynamic topology of interconnections and automatic administration for setting of the network. Mathematically, an Ad hoc network is a Graph $G=\{N, E\}$, which consists of set of nodes $\mathrm{N}$ and set of edges E. Each mobile host can be node of the graph. Each edge e $\epsilon$ E is formed by two nodes within the radio range, it can be unidirectional or bi-directional, e $\epsilon \mathrm{E}$ is dynamic and changes with time as the mobile nodes in the Ad hoc network freely move around. The topology of the Ad hoc network can be arbitrary at any time. Due to dynamic topology of an Ad hoc network, the nodes in the network have to update their routing information automatically and instantly. Routing in Ad hoc networks is different and challenging when compared to routing in conventional networks.

Routing protocols in Ad hoc networks can be clarified into three categories namely, proactive, on-demand also called as reactive, and hybrid protocols [8] \& [9].

In proactive routing protocols, each node in the network maintains a route to every other node in the network. The routing information in maintained in a table known as routing table (RT) and periodically or when every there is a change in topology of network, the nodes update their routing table by a protocol known as Routing Information Protocol (RTP). The proactive routing protocols are also known as table driven protocols. DSDV, STAR are examples of proactive protocols.

In reactive protocols, node in the network discovers a route to another node only when it has to send data packets to some destination, it checks its route table to determine whether it has route. If no route exists, it performs a route discovery procedure to find a path to the destination. Hence, route discovery becomes on-demand DSR, AODV are on-demand routing protocols.

In hybrid protocols, both the characteristics of proactive/tabledriven and reactive/on-demand are followed. Hybrid protocols are basically distance vector i.e., proactive but contain many features of on-demand i.e., reactive protocols. ZRP are the hybrid routing protocols. The objective of HRP is to find optimal path between two nodes with minimal usage of battery power, minimum delay, using less memory and less processing power.

In application, reactive/on-demand routing are widely used and implemented. The main problem in on-demand routing protocols is discovery of multiple paths between a source and destination node. An effective mechanism is needed to eliminate sparse paths and select the best path from the discovered routes.

In this paper we propose a new reactive/on-demand routing protocol (TSBR) for Ad hoc networks. TSBR is an ondemand routing protocol that solves the problem of multiple paths between two nodes through a time stamp values.

In TSBR each discovered route is assigned a time stamp value and among the discovered routes the route with minimum time stamp value is selected to send data packets.

The section 2 of the paper holds the description of TSBR, the simulation of TSBR is given in section 3. Analysis of the results is given in section 4 and conclusion in section 5 .

\section{TSBR}

TSBR is an on-demand routing protocol for Ad hoc networks. In TSBR each discovered route is assigned a time stamp value and stored as routing information in route table. Routing in TSBR is in two phases viz. a) Route discovery and b) Route maintenance.

\subsection{Route Discovery}

When a source node has to send data packets to a destination node, it searches its route table to find if the route already exists. If no route exists then the source node initiates a route discovery process by broadcasting RREQ (Route Request) 
packet to all its neighbors. The RREQ packet contains the ID of source node, ID of destination node, monotonically increasing value Time to Live (TTL) a time stamp value $T_{r q}$, next hop field.

When a RREQ reaches a mobile node and if it is not the destination node, it add its ID to the next hop field and forwards/multicasts to its neighbors, and broadcasts a route Reply (RREP) packet to the sender along the reverse path in next hop field. This continues until the RREQ packet reaches the destination node or the TTL reaches a threshold value. If the TTL reaches the threshold value, then the next immediate node on the network discards (RREQ) packet and sends Route Error (RERR) packet to the source node along reverse path.

If the RREQ packet reaches the destination node, it sends a RREP packet along the reverse path. RREP packet contains the ID of source node, ID of destination node, monotonically increasing TTL, Timestamp value $\mathrm{T}_{\mathrm{rr}}$. When source node receives a RREP packet, it updates it route table using update algorithm

\subsubsection{Update Algorithm}

Begin

For every RREP packet received do

Begin

Calculate $T^{\prime}=T_{r r}-T_{r q}$

If there is no entry in the route table for the destination node $\mathrm{D}$,

then

add entry to the route table with destination field as D, next hop field as advertised neighbor node $\mathrm{N}$ and time stamp T'.

else

If the next-hop field in route table and RREP packet are same

Then

\section{If $\mathrm{T}>\mathrm{T}$}

Then

Update $\mathrm{T}=\mathrm{T}^{\prime}$

Else

Break

Else

$$
\begin{aligned}
& \text { If } \mathrm{T}>\mathrm{T}^{\prime} \\
& \text { Then }
\end{aligned}
$$

Update route table with nexthop as the advertised neighbor node $\mathrm{N}$ and Time stamp as T'

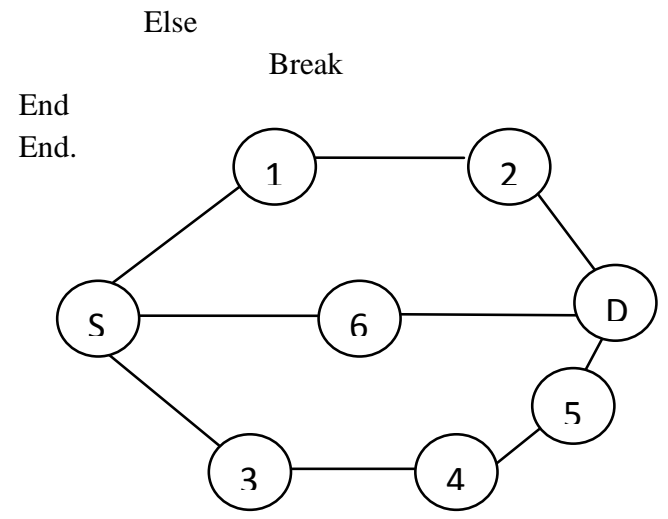

Fig. 1 Network Topology
A simple example is illustrated in Fig. 1. Three possible paths $\mathrm{S} \rightarrow>1->2->\mathrm{D}, \mathrm{S}->3 \rightarrow 4->5 \rightarrow \mathrm{D} \& \mathrm{~S} \rightarrow 5 \rightarrow \mathrm{D}$ are multiple paths between source node $S$ and the destination node $D$.

The route table of s will finally hold the route information for route $S \rightarrow 6 \rightarrow D$ for the path between source node $S$ to destination node $\mathrm{D}$ as the time stamp value of this path is minimum.

\section{Packets received}

$\mathrm{PDR}=$

\section{Packets generated}

\subsection{Route maintenance}

The route once discovered between a source node and destination node is used as for as is needed. But, in the mean time if source node $S$, moves it deletes the entries in its route table and reinitiates a Route Discovery Processes. If destination/other node move, it sends this information to the source node through RERR packet. Source node upon receiving a RERR packet for a destination node, it stops sending data packets to the destination node, remove the entry of destination node from its route table, if already exists and re- initiates route discovery to the destination node.

\section{SIMULATION}

The proposed protocol TSBR was simulated in NS -2 network simulator [10] \& [11].

\subsection{Simulation Parameters}

\begin{tabular}{|l|l|}
\hline Parameter & Value \\
\hline Simulator & NS-2 \\
\hline Node movement & Random Way Point \\
\hline MAC protocol & 802.11 \\
\hline Play ground size & $1000 \mathrm{~m} \mathrm{X} \mathrm{1000} \mathrm{m}$ \\
\hline No. of nodes & 20 \\
\hline Node speed & 1 to $20 \mathrm{~m} / \mathrm{s}$ \\
\hline Bandwidth & 2 Mbps \\
\hline Data Packet Size & 1000 bytes \\
\hline Traffic Type & CBR \\
\hline
\end{tabular}

Table 1 Simulation set up

The performance metrics used in the simulation are Packet Delivery Ratio and Average End-to-End delay. 


\subsection{Packet Delivery Ratio (PDR)}

Packet delivery ratio (PDR) [7] is the number of packets received by the destination nodes to the number of packets generated by the source node.

\subsection{Average End-to-End Delay}

Average End-to-End delay [2] [5] \& [6] is the average of the time delay of packets from source nodes to destination nodes.

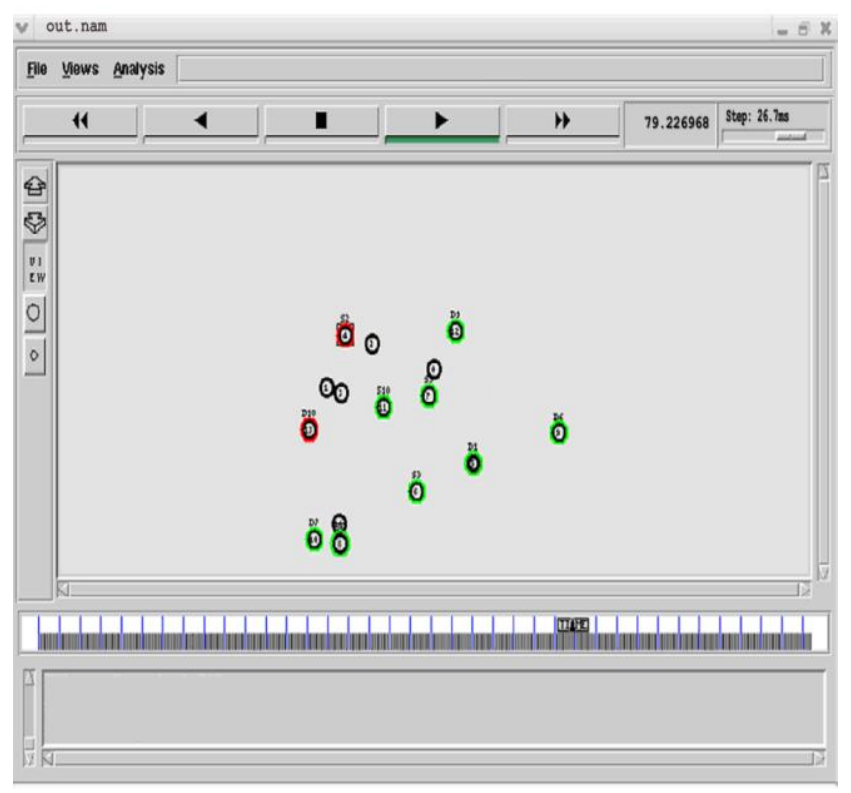

Fig. 2 Sample Screen Shot

\section{RESULTS AND ANALYSIS}

The PDR was found to be 0.87 at pause time 700 and Avg. End-to-End delay was found to be 900 at pause time 600. The PDR and Avg. End-to-End Delay of TSBR are optimal. Due to low mobility at pause time 600 the Avg. End-to-End was minimum and PDR was max. at pause time 700 due to low data loss.

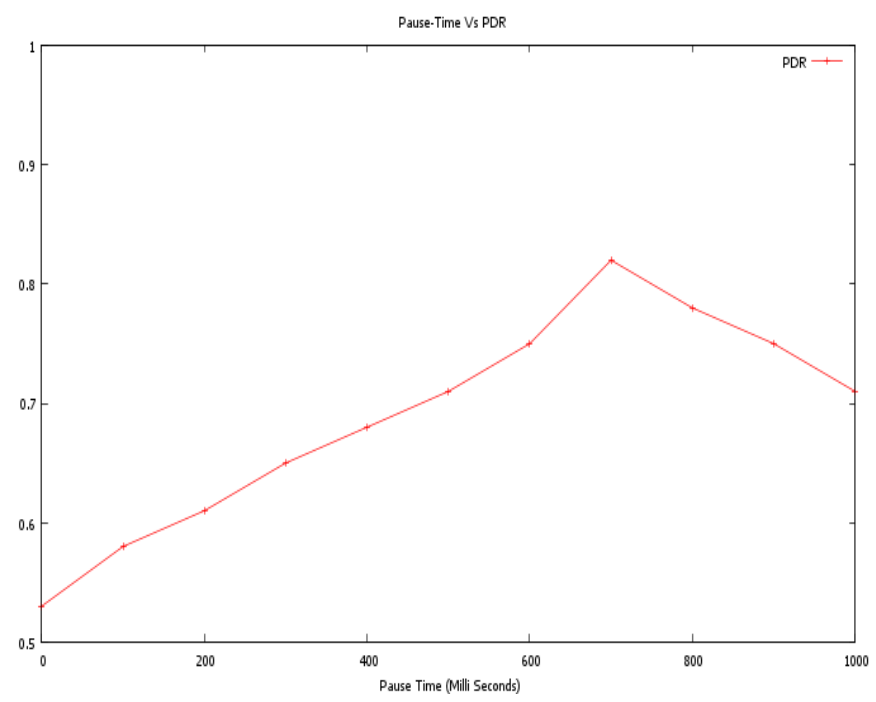

Fig . 3 Pause time Vs PDR

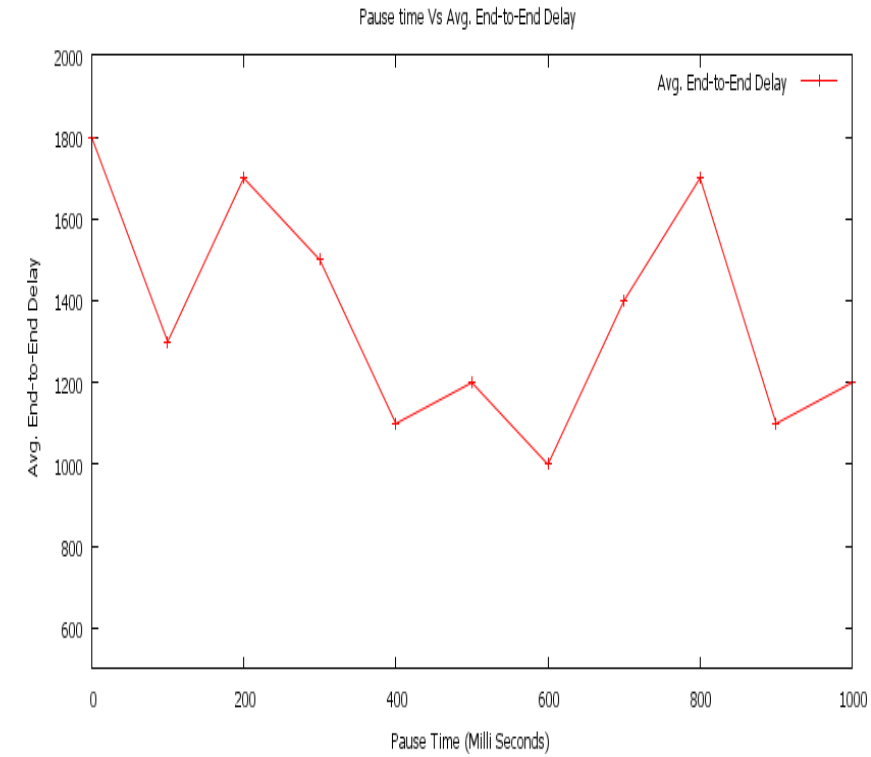

Fig. 4 Pause time Vs End-to-End Delay

\section{CONCLUSION}

In this paper, we have proposed TSBR to improve the performance of routing protocols in Ad hoc networks. TSBR is an on-demand/reactive routing protocol that uses the Time Stamp values, to eliminate the sparse routes between a source and destination nodes. The route selected to send data packets in the route with minimum time stamp, means the path that takes less time to transmit packets between source and destination nodes.

The TSBR can also be applied to DTN with some modifications and up gradations.

\section{REFERENCES}

[1] Charles E Perkins and Elizabeth M Royer, "Ad Hoc OnDemand Distance Vector Routing", in Proceedings of the $2^{\text {nd }}$ IEEE Workshop on Mobile Computing Systems and Applications, Feb. 1999, pp 90-100.

[2] Z.Alexander, "Performance Evaluation of AODV Routing Protocol:Real-Life Measurements", SCC, June 2003.

[3] David B Johnson, David A Maltz, Yin Chun Hu, “ The Dynamic Source Routing Protocol for Mobile Ad Hoc Networks(DSR)", Internet draft,draftietfManetdsr09.txt, April, 2003.

[4] Charles E Perkins, Elizabeth M, Belding Royer and Samir R Das, "Ad Hoc On-demand Distance Vector (AODV) Routing”, Mobile Ad-hoc Networking Group, Internet draft, February 2003.

[5] P Michiardi and R Molva, "Simulation based Analysis of Security Exposures in Mobile ad-hoc Networks", European Wireless 2002 Conference, 2002. 
[6] Josh Broch, David A Maltz, David B Johnson, Yin Chun $\mathrm{Hu}$ and Joreta Jetcheva A, "Performance Comparision of Multi-Hop Wireless Ad-hoc Network Routing Protocols", Proceedings of the Fourth Annual ACM/IEEE International Conference on Mobile Computing and Networking (MobiCom '98), pages 8597, October 1998, ACM.

[7] Zygmunt J Haas, " A New Routing Protocol for the Reconfigurable Wireless Networks", proceedings of the $6^{\text {th }}$ International Conference on Universal Personal Communications, pages 562-566, IEEE, October 1997.
[8] David B Johnson, “ Routing in Ad Hoc Networks of Mobile Hosts", Proceedings of the IEEE Workshop on Mobile Conputing Systems and Applications, pages 158163, IEEE Computer Society, December 1994.

[9] Kristoffer Clyde Magsino and H Srikanth Kamath, "Simulations of Routing Protocols of Wireless Sensor Networks", World Academy of Science, Engineering and Technology 50, pages 211-214, 2009.

[10] NS-2Simulator, http://www.isi.edu/nsnam/ns/

[11] The Network simulator NS-2 tutorial home page, http://www.isi.edu/nsnam/ns/tutorial/index.html 